\title{
Different intensities of basketball drills affect jump shot accuracy of expert and junior players
}

\author{
Giuseppe Marcolin $^{1}{ }^{1}$, Nicola Camazzola ${ }^{2}$, Fausto Panizzolo ${ }^{3}$, Davide Grigoletto $^{1}$, Antonio Paoli $^{\text {Corresp. }}{ }^{1}$ \\ 1 Department of Biomedical Sciences, University of Padova, Padova, Italy \\ 2 School of Human Movement Sciences, University of Padova, Padova, Italy \\ 3 John A. Paulson School of Engineering and Applied Sciences, Harvard University, Cambridge, Massachusetts, United States \\ Corresponding Author: Antonio Paoli \\ Email address: antonio.paoli@unipd.it
}

Background. In basketball a maximum accuracy at every game intensity is required while shooting. The aim of the present study was to investigate the acute effect of three different drill intensity simulation protocols on jump shot accuracy in expert and junior basketball players. Materials \& Methods. Eleven expert players (age $26 \pm 6 \mathrm{yrs}$, weight $86 \pm 11 \mathrm{~kg}$, height $192 \pm 8 \mathrm{~cm}$ ) and ten junior players (age $18 \pm 1 \mathrm{yrs}$, weight $75 \pm 12 \mathrm{~kg}$, height $184 \pm 9 \mathrm{~cm}$ ) completed three series of twenty jump shots at three different levels of exertion. Counter Movement Jump (CMJ) height was also measured after each series of jump shots. Exertion's intensity was induced manipulating the basketball drills. Heart rate was measured for the whole duration of the tests while the rating of perceived exertion (RPE) was collected at the end of each series of shots. Results. Heart rate and rating of perceived exertion (RPE) were statistically different in the three conditions for both expert and junior players. CMJ height remained almost unchanged in both groups. Jump shot accuracy decreased with increasing drills intensity both in experts and junior players. Expert players showed higher accuracy than junior players for all the three levels of exertion ( $83 \%$ vs $64 \%, p<0.001 ; 75 \%$ vs $57 \%, p<0.05 ; 76 \%$ vs $60 \%, p<0.01$ ). Moreover, for the most demanding level of exertion, experts showed a higher accuracy in the last ten shots compared to the first ten shots ( $82 \%$ vs $70 \%, p<0.05)$. Discussion. Experts coped better with the different exertion's intensities, thus maintaining a higher level of performance. The introduction of technical short bouts of high-intensity sport-specific exercises into skill sessions should be proposed to improve jump shot accuracy during matches 


\section{Different intensities of basketball drills affect jump shot accuracy of expert and junior 2 players.}

4 Giuseppe Marcolin $\mathrm{PhD}^{1}$, Nicola Camazzola BSc${ }^{2}$, Fausto A. Panizzolo $\mathrm{PhD}^{3}$, Davide Grigoletto $5 \quad \mathrm{BSc}^{1}$ and Antonio Paoli BSc, $\mathrm{MD}^{1}$

$6 \quad{ }^{1}$ Department of Biomedical Sciences, University of Padova, Padova, Italy

$7 \quad 2$ School of Human Movement Sciences, University of Padova, Padova, Italy

$8{ }^{3}$ John A. Paulson School of Engineering and Applied Sciences, Harvard University, Cambridge, $9 M A, U S A$

11 Corresponding author:

12 Antonio Paoli, M.Sc, MD

13 Department of Biomedical Science, University of Padova,

14 Via Marzolo 3, 35131 Padova, Italy

15 Phone: 00390498275318

16 Fax: 00390498275301

17 E-mail: antonio.paoli@unipd.it 


\section{Abstract}

25 Background. In basketball a maximum accuracy at every game intensity is required while 26 shooting. The aim of the present study was to investigate the acute effect of three different drill intensity simulation protocols on jump shot accuracy in expert and junior basketball players.

Materials \& Methods. Eleven expert players (age $26 \pm 6$ yrs, weight $86 \pm 11 \mathrm{~kg}$, height $192 \pm 8 \mathrm{~cm}$ ) and ten junior players (age $18 \pm 1 \mathrm{yrs}$, weight $75 \pm 12 \mathrm{~kg}$, height $184 \pm 9 \mathrm{~cm}$ ) completed three series of twenty jump shots at three different levels of exertion. Counter Movement Jump (CMJ) height was also measured after each series of jump shots. Exertion's intensity was induced manipulating the basketball drills. Heart rate was measured for the whole duration of the tests while the rating of perceived exertion (RPE) was collected at the end of each series of shots.

Results. Heart rate and rating of perceived exertion (RPE) were statistically different in the three conditions for both expert and junior players. CMJ height remained almost unchanged in both groups. Jump shot accuracy decreased with increasing drills intensity both in experts and junior exertion ( $83 \%$ vs $64 \%, p<0.001 ; 75 \%$ vs $57 \%, p<0.05 ; 76 \%$ vs $60 \%, p<0.01)$. Moreover, for the most demanding level of exertion, experts showed a higher accuracy in the last ten shots compared to the first ten shots $(82 \%$ vs $70 \%, \mathrm{p}<0.05)$.

41 Discussion. Experts coped better with the different exertion's intensities, thus maintaining a

42 higher level of performance. The introduction of technical short bouts of high-intensity sport- 
43 specific exercises into skill sessions should be proposed to improve jump shot accuracy during 44 matches.

\section{Introduction}

Basketball is a common sport where conditioning and fatigue affect performance (Erculj

\& Supej, 2009). Very specific physiological requirements are associated with this sport, with

mean heart rate during live time of $169 \pm 9$ beats per minute and mean blood lactate concentration of $6.8 \pm 2.8 \mathrm{mmol} \cdot \mathrm{L}^{-1}$ (McInnes, Carlson, Jones, \& McKenna, 1995). Together with a high level of fitness, maximum accuracy is required while performing specific motor tasks such as shooting at a target (Erculj \& Supej, 2009). fatigue as well as physical exertions at different intensities on several aspects of basketball. Ahmed (Ahmed, 2013) investigated the effect of upper extremity fatigue on grip strength and passing accuracy, showing a significant decrease of both these variables after the fatigue protocol

57 administered. Similarly, it has been found that a detriment in the passing performance among novice and expert basketball players followed a high intensity total body fatigue protocol (Lyons, Al-Nakeeb, \& Nevill, 2006). The effect of fatigue on 3 point-shooting has been investigated

60 from a biomechanical point of view analyzing the position of the release arm and shoulder girdle

61 showing that all the measured angles decreased drastically as a consequence of the heavy level of 62 fatigue (Erculj \& Supej, 2009). Three point-shooting accuracy has also been investigated after 63 two different resistance circuit training protocols showing a reduction in accuracy only after the 64 most intensive protocol (Freitas, Calleja-González, Alarcón, \& Alcaraz Ramón, 2016).

65 Conversely, free throw shooting analysis demonstrated that fatigue did not affect kinematics and 
66 that shooting technique was the same during successful and unsuccessful shots (Uygur, Goktepe,

67 Ak, Karabörk, \& Korkusuz, 2010). Similarly repeated sprint test performance indices remained 68 unchanged after full time or even improved after half time in comparison with the same indices 69 performed after the warm up (Meckel, Gottlieb, \& Eliakim, 2009).

In the evaluation of fatigue on sport skill performance, it has been shown that a key point

71 is the employment of sport-specific training methods to induce fatigue (P. R. Davey, Thorpe, \&

72 Williams, 2002; P. Davey, Thorpe, \& Williams, 2003; Vergauwen, Spaepen, Lefevre, \& Hespel, 1998). To the best of our knowledge, while in water polo (Royal et al., 2006), tennis (Lyons, AlNakeeb, Hankey, \& Nevill, 2013) and soccer (Rampinini et al., 2008) accuracy was evaluated employing these sport-specific methods, while in basketball passing (Ahmed, 2013; Lyons et al., 2006) and shooting (Freitas et al., 2016) accuracy was quantified after standard strength training exercises.

Therefore the purpose of the present study was to analyze the effect of three different exertion's intensities induced with sport-specific drills on the jump shot accuracy and jump

height of expert and junior basketball players. Our hypothesis was to find in both groups a

81 decrease of jump shot accuracy and jump height as exertion increased. Moreover we expected 82 expert players to better cope with the three exertion's intensities compared to junior players 83 because of their higher level of expertise.

84 Materials \& Methods

85

86

87 88

Participants group included 11 expert players (age $26 \pm 6$ yrs, weight $86 \pm 11 \mathrm{~kg}$, height $192 \pm 8 \mathrm{~cm}$ ) and the second 10 junior players (age $18 \pm 1 \mathrm{yrs}$, weight $75 \pm 12 \mathrm{~kg}$, height $184 \pm 9 \mathrm{~cm}$ ). Experts trained four 
89 times per week while junior players three times. Inclusion criteria included at least 10 years of

90 competitive basketball experience for expert players and 5 years for junior players, regular

91 participation to official matches and lack of any muscle and tendon pathologies to upper and

92 lower limbs at the time of the study. A detailed description of the experimental procedures was

93 given to each participant which provided an informed consent prior to testing. The study was

94 approved by the ethical committee of the Department of Biomedical Sciences, University of 95 Padova (number HEC-DSB11/16).

\section{Experimental protocol}

Each participant was asked to perform three series of twenty jump shots at three different exertion's intensities. A 15 minute standardized warm up, consisting of 10 minutes of aerobic conditioning and 5 minutes of free throw shots, took place before the three series of jump shots. In the low intensity protocol (LIP) the participant stayed with feet on the free throw line, received the ball from a teammate positioned under the basket, and performed the jump shot. The teammate moved to regain the ball to perform the successive pass. This procedure was repeated until the twenty jump shots were completed. In the moderate intensity protocol (MIP) each participant started the series from the half-court line. Then he ran slowly to one of the two cones placed on the 3 point line, by the side of the extension of the long sides of the 3 seconds area. After reaching the cone, he performed a side cutting maneuver towards the free throw line where he received the ball from a teammate and performed the jump shot. Subsequently the participant returned to the half-court line walking and started again. This course was repeated until the twenty jump shots were completed. Unlike the moderate intensity, in the high intensity protocol (HIP) participants sprinted towards the cone and, after the side cutting maneuver, towards the free throw line. The return to the half-court line was done half walking and half slowly running. 
112 In both moderate and high intensity protocols each participant had to choose one of the two

113 cones and then perform the side cutting maneuver on the same cone for all the trials. The rest

114 among the three series was set to 4 minutes. Before the beginning of the first series of jump shots

115 and immediately after the moderate and high intensity series three counter movement jumps

116 (CMJ) were collected for each athlete by means of a Bosco conductance mat (Globus Italia,

117 Treviso, Italy) which estimates the height of the jump measuring the flight time. For the whole

118 duration of the tests participants wore a chest band which recorded the heart rate at a sampling

119 rate of $1 \mathrm{~Hz}$ (Garmin, Kansas City, USA). In order to calculate Karvonen heart rate reserve

120 (\%HRR) (Karvonen, Kentala, \& Mustala, 1957) at each intensity of induced exertion, resting

121 heart rate was assessed by each participant when they woke up in the morning for the two days

122 prior to the tests. At the end of each set of shots the rating of perceived exertion (RPE) was

123 recorded by means of a 6-20 Borg scale (Haile, Gallagher, \& Robertson, 2015). A schematic of

124 the experimental setup is reported in figure 1.

\section{Statistical analysis}

126 One way repeated measurements analysis of variance (ANOVA) was used to compare the

127 three exertion's intensities. Significant level was set at $p<0.05$. If the statistical significance was

128 reached, Tukey's multiple comparison test was employed. Unpaired t-test was used to compare

129 expert and junior players at every level of induced exertion. Data analysis was performed with

130 the software package GraphPad Prism version 4.00 for Windows (GraphPad Software, San

131 Diego California USA). Statistical effect size was calculated with the G*Power 3.1.5 software

132 (Faul, Erdfelder, Lang, \& Buchner, 2007). 


\section{Results}

134 Significant differences among the exertion's intensities reached during the three sets of

135 jump shots for the two groups of athletes were reported by the statistical analysis (Table 1).

136 Among experts, heart rate values were lower in LIP with respect to MIP ( $<<0.01$, ES: 1.00) and

137 HIP $(p<0.01$, ES: 3.06) and the rate of perceived exertion was lower in LIP with respect to MIP

$138(\mathrm{p}<0.01, \mathrm{ES}: 1.50)$ and HIP $(\mathrm{p}<0.01$, ES: 4.00). Among junior players, heart rate values were

139 lower in LIP with respect to MIP ( $p<0.01$, ES: 0.75) and HIP ( $p<0.01$, ES: 2.19$)$ and the rate of

140 perceived exertion was lower in LIP with respect to MIP ( $p<0.05$, ES: 1.09) and HIP $(\mathrm{p}<0.01$,

141 ES: 3.56). In the LIP condition, RPE was higher for juniors players in comparison with experts

142 players $(\mathrm{p}<0.05$, ES: 1.25). MIP and HIP conditions were perceived equally demanding by

143 experts and junior players with no statistically significant differences among RPE values. Shot

144 accuracy of expert players was 83\% (LIP), 75\% (MIP) and 76\% (HIP), while for the group of

145 junior players it was 64\% (LIP), 57\% (MIP), 60\% (HIP). Conversely, jump height was very

146 similar for junior players and expert players (Table 1). Moreover, a statistically significant

147 increase of expert players' jump height after HIP with respect to warm up was found ( $\mathrm{p}<0.01$;

$148 \mathrm{ES}=0.58$ ). The analysis of jump shot accuracy between expert and junior players revealed a

149 better proficiency among expert players at every intensity of induced exertion (LIP: p<0.001, ES:

150 1.74; MIP: $p<0.05$, ES: 1.03; HIP: $\mathrm{p}<0.01$, ES: 1.37), as reported in figure 2. A further analysis

151 compared the effect of the three protocols on jump shot accuracy of the first ten shots with the

152 last ten shots. Expert players showed a decrease of accuracy only between LIP and HIP ( $\mathrm{p}<0.05$,

153 ES: 1.03) while junior players showed no statistically significant differences in the first ten shots.

154 On the other hand, variation of the accuracy of the last ten shots was not statistically significant

155 neither in expert nor in junior players. Moreover, expert players showed a higher accuracy

156 comparing the second ten shots with the first ten shots only in the HIP condition ( $p<0.05$, ES: 
157 0.73). No statistically significant differences were detected among junior players. All the results

158 relative to the first and to the last ten shots for both groups are reported in Table 2.

\section{Discussion}

160 To assess the effect of different exertion's intensities on sport-specific skills it is essential

161 to employ sport-specific protocols for an ecological validity of the experimental results (Lyons et

162 al., 2006; Royal et al., 2006). In the present study three different exertion's intensities (LIP, MIP

163 and HIP) were induced by means of basketball drills such as sprinting, cutting maneuvers and

164 passes allowing to investigate jump shot accuracy in expert and junior players. A decrease in

165 shot accuracy was detected for both groups similarly to previous investigations where technical

166 skills deteriorated as a consequence of fatigue (Freitas et al., 2016; Lyons et al., 2006; Rampinini

167 et al., 2008). Conversely, the results of the present study showed no differences in jump height

168 after the three protocols. Similar results were obtained investigating repeated sprint tests at

169 different game stages (Meckel et al., 2009) underling the importance of an intense warm up to

170 improve sprint performance in the initial phases of a basketball match. Despite the RPE and HR

171 results, in the present work the three different exertion's intensities proposed cannot be

172 considered as fatigue promoters since a decrease of the jump performance was not detected after

173 MIP and above all HIP. However, the intensities proposed with regards to HIP allowed to

174 investigate how the jump shot accuracy varied in a contest similar to a real match play.

175 Therefore our results supported the suggestion of training at level of exertion similar to

176 those recorded during competitive games (Erculj \& Supej, 2009; Freitas et al., 2016; Lyons et al.,

1772013 , 2006) together with the recommendation to employ sport-specific drills to increase the

178 exertion's intensity (Lyons et al., 2006; Royal et al., 2006). The decrease in jump shot accuracy

179 was indeed more marked for both groups comparing LIP with HIP rather than MIP compared to 
180 HIP. Thus, an increased number of high intensity skill sessions should aim to improve the shot

181 accuracy at those exertion's intensities representative of a match. However, during the

182 introduction of these technical short bouts of high-intensity exercise into skill sessions the

183 technique should be monitored to assure a correct execution (Lyons et al., 2013).

184 The comparison between expert and young players showed a higher accuracy in the

185 expert group at each exertion's intensity. Our results are comparable with previous

186 investigations on basketball (Lyons et al., 2006) and tennis (Lyons et al., 2013), showing how

187 expert players can cope better with higher exertion's intensities, thus maintaining a higher level

188 of performance. This could be due to the fact that technical and motor patterns are strongly

189 formed in experts and that they can adjust motor coordination strategies as a reaction to induced

190 exertion better than young players (Aune, Ingvaldsen, \& Ettema, 2008). Therefore it is clear

191 again how with young players the introduction of technical short bouts of high-intensity exercise

192 into skill sessions should be carefully implemented to avoid technique alterations as much as

193 possible (Lyons et al., 2013).

194 An additional interesting point relative to HIP is the higher shot accuracy recorded by

195 expert players in the last ten shots with respect to that recorded in the first ten shots. Since

196 athletes begin the HIP bout after a 4-minute recovery (see figure 1), only in the second part of

197 the bout heart rate values were similar to those recorded in a real match (Erculj \& Supej, 2009).

198 In fact expert player mean heart rate was $143 \pm 9$ beats $\cdot \mathrm{min}^{-1}$ during the first ten shots and $165 \pm 9$

199 beats $\cdot \mathrm{min}^{-1}$ in the last ten shots. This condition, together with the employment of basketball-

200 specific tasks, could have created sensory states similar to those experienced in contest inducing

201 participants to use the same processes responsible for their expertise in match-play (Royal et al.,

202 2006). For the same reason junior players could have maintained their shot accuracy in the last 
203 ten shots in comparison with that recorded in the first ten shots. In fact their mean heart rate was

$204153 \pm 10$ beats $\cdot \min ^{-1}$ and $176 \pm 8$ beats $\cdot \min ^{-1}$ during the first and the last ten shots respectively. To

205 this extent, we can hypothesize that in the last ten shots of HIP the decrease of shot proficiency

206 due to their lower ability in coping with high exertion's intensity was counterbalanced by the

207 sensory states typical of a match-play they experienced.

208 Jump shot accuracy results referred to the highest exertion's intensity excluded a possible 209 relationship between shot proficiency and mental fatigue (Boksem, Meijman, \& Lorist, 2005;

210 Faber, Maurits, \& Lorist, 2012). In fact if mental fatigue would have been induced, the shot

211 accuracy of the last ten shots in the HIP should have been the worst. Moreover it has been shown

212 how mental fatigue affected marksmanship judgment in soldiers but not their shot accuracy

213 (Head et al., 2017).

214 On the importance of the exertion's intensity on performance an additional aspect

215 deserves consideration. Previous literature (Meckel et al., 2009) reported that an intensive warm

216 up is needed to increase repeated sprint performance at the initial phases of the match. Our

217 findings on the accuracy during HIP, comparing the first ten shots with the second ten shots, are

218 in agreement with this theory. When heart rate was stabilized at a high value, accuracy increased

219 in experts and stayed constant in junior players. Therefore, an intensive warm-up could be useful

220 to activate a game-specific arousal since the initial phases of the match. Moreover short intensive

221 exercises could be proposed for the cases in which a player comes off the bench to reactivate this

222 game-specific arousal entering in the court.

\section{Conclusions}

224 Our study showed how expert basketball players coped better with different exertion's 225 intensities with respect to junior players, thus maintaining a higher level of jump shot 
226 proficiency. Moreover, expert players showed at HIP the best shot accuracy when heart rate was

227 high in the last ten shots. This high exertion's intensity together with the employment of sport 228 specific drills could have induced participants to activate during training the same processes 229 responsible for their expertise in real match-play. Therefore our findings could be of practical 230 interest for coaches to improve the efficacy of technical skill sessions during training and warm

231 up before matches. However the introduction of these high-intensity technical exercises into skill 232 sessions should be carefully monitored to avoid technique alterations. Further studies are 233 required to investigate how the exertion's intensity of different sport-specific protocols could be 234 used to activate game-specific arousal on athletes with distinct levels of expertise without 235 altering the technical execution of the jump shot.

\section{References}

237

238

239

240

241

242

243

Ahmed, T. (2013). The Effect of Upper Extremity Fatigue on Grip Strength and Passing Accuracy in Junior Basketball Players. Journal of Human Kinetics, 37(1), 71-79. https://doi.org/10.2478/hukin-2013-0027

Aune, T. K., Ingvaldsen, R. P., \& Ettema, G. J. C. (2008). Effect of physical fatigue on motor control at different skill levels. Perceptual and Motor Skills, 106(2), 371-386. https://doi.org/10.2466/pms.106.2.371-386

Boksem, M. A. S., Meijman, T. F., \& Lorist, M. M. (2005). Effects of mental fatigue on attention: An ERP study. Cognitive Brain Research, 25(1), 107-116. https://doi.org/10.1016/j.cogbrainres.2005.04.011

Davey, P. R., Thorpe, R. D., \& Williams, C. (2002). Fatigue decreases skilled tennis performance. Journal of Sports Sciences, 20(4), 311-318. https://doi.org/10.1080/026404102753576080

Davey, P., Thorpe, R., \& Williams, C. (2003). Simulated tennis matchplay in a controlled 
250

251

252

253

254

255

256

257

258

259

260

261

262

263

264

265

266

267

268

269

270

271

272

273

274

275

276

environment. Journal of Sports Sciences, 21(6), 459-467.

https://doi.org/10.1080/0264041031000101926

Erculj, F., \& Supej, M. (2009). Impact of fatigue on the position of the release arm and shoulder girdle over a longer shooting distance for an elite basketball player. Journal of Strength and Conditioning Research, 23(3), 1029-1036. https://doi.org/doi:

10.1519/JSC.0b013e3181a07a27.

Faber, L. G., Maurits, N. M., \& Lorist, M. M. (2012). Mental Fatigue Affects Visual Selective Attention. PLoS ONE, 7(10), e48073. https://doi.org/10.1371/journal.pone.0048073

Faul, F., Erdfelder, E., Lang, A.-G., \& Buchner, A. (2007). G*Power 3: a flexible statistical power analysis program for the social, behavioral, and biomedical sciences. Behavior Research Methods, 39(2), 175-191. https://doi.org/10.3758/BF03193146

Freitas, T. T., Calleja-González, J., Alarcón, F., \& Alcaraz Ramón, P. E. (2016). Acute Effects of Two Different Resistance Circuit Training Protocols on Performance and Perceived Exertion in Semiprofessional Basketball Players. Journal of Strength and Conditioning Research, 30(2), 407-414. https://doi.org/10.1519/JSC.0000000000001123

Haile, L., Gallagher, M. J., \& Robertson, R. J. (2015). Perceived exertion laboratory manual: From standard practice to contemporary application. Perceived Exertion Laboratory Manual: From Standard Practice to Contemporary Application. https://doi.org/10.1007/978-1-4939-1917-8

Head, J., Tenan, M. S., Tweedell, A. J., LaFiandra, M. E., Morelli, F., Wilson, K. M., ... Helton, W. S. (2017). Prior Mental Fatigue Impairs Marksmanship Decision Performance. Frontiers in Physiology, 8, 680. https://doi.org/10.3389/fphys.2017.00680

Karvonen, M. J., Kentala, E., \& Mustala, O. (1957). The effects of training on heart rate; a longitudinal study. Annales Medicinae Experimentalis et Biologiae Fenniae, 35(3), 307-15.

Lyons, M., Al-Nakeeb, Y., Hankey, J., \& Nevill, A. (2013). The effect of moderate and highintensity fatigue on groundstroke accuracy in expert and non-expert tennis players. Journal of Sports Science and Medicine, 12(2), 298-308. 
277 Lyons, M., Al-Nakeeb, Y., \& Nevill, A. (2006). The impact of moderate and high intensity total 278 body fatigue on passing accuracy in expert and novice basketball players. Journal of Sports 279 Science \& Medicine, 5(2), 215-227. https://doi.org/10.1519/JSC.0b013e3181a4e7f0

280 McInnes, S., Carlson, J., Jones, C., \& McKenna, M. (1995). The physiological load imposed on 281 282

Meckel, Y., Gottlieb, R., \& Eliakim, A. (2009). Repeated sprint tests in young basketball players at different game stages. European Journal of Applied Physiology, 107(3), 273-279. https://doi.org/10.1007/s00421-009-1120-8

Rampinini, E., Impellizzeri, F. M., Castagna, C., Azzalin, A., Bravo, D. F., \& Wisløff, U. (2008). Effect of match-related fatigue on short-passing ability in young soccer players. Medicine and Science in Sports and Exercise, 40(5), 934-942. https://doi.org/10.1249/MSS.0b013e3181666eb8

Royal, K., Farrow, D., Mujika, I., Halson, S., Pyne, D., \& Abernethy, B. (2006). The effects of fatigue on decision making and shooting skill performance in water polo players. Journal of Sports Sciences, 24(8), 807-815. https://doi.org/10.1080/02640410500188928

Uygur, M., Goktepe, A., Ak, E., Karabörk, H., \& Korkusuz, F. (2010). The Effect of Fatigue on the Kinematics of Free Throw Shooting in Basketball. Journal of Human Kinetics, 24(24), 51-56. https://doi.org/10.2478/v10078-010-0019-0

Vergauwen, L., Spaepen, A. J., Lefevre, J., \& Hespel, P. (1998, August). Evaluation of stroke performance in tennis. Medicine and Science in Sports and Exercise. https://doi.org/10.1097/00005768-199808000-00016 
300 Figure captions

301 Figure 1. Schematic representation of the experimental protocol.

302 Figure 2. Baskets scored in the LIP (A), MIP (B) and HIP (C) conditions: comparison between

303 experts and young players $\left(* \mathrm{p}<0.05 ;{ }^{*} \mathrm{p}<0.01 ; * * * \mathrm{p}<0.001\right)$.

304

305

306

307

308

309

310

311

312

313

314

315

316

317

318

319

320

321

322 


\section{Table captions}

326 Table 1. Heart rate, rate of perceived exertion (RPE), jump shot accuracy and jump height

327 recorded immediately after each fatigue protocol. Data are presented as mean \pm s. For both 328 groups: * different from low intensity fatigue protocol (LIP); $\S$ different from moderate intensity 329 fatigue protocol (MIP).

330 Table 2. Baskets scored for each level of induced fatigue. Experts: * Different from 1-10 series. 
Figure 1

Experimental protocol

Schematic representation of the experimental protocol.

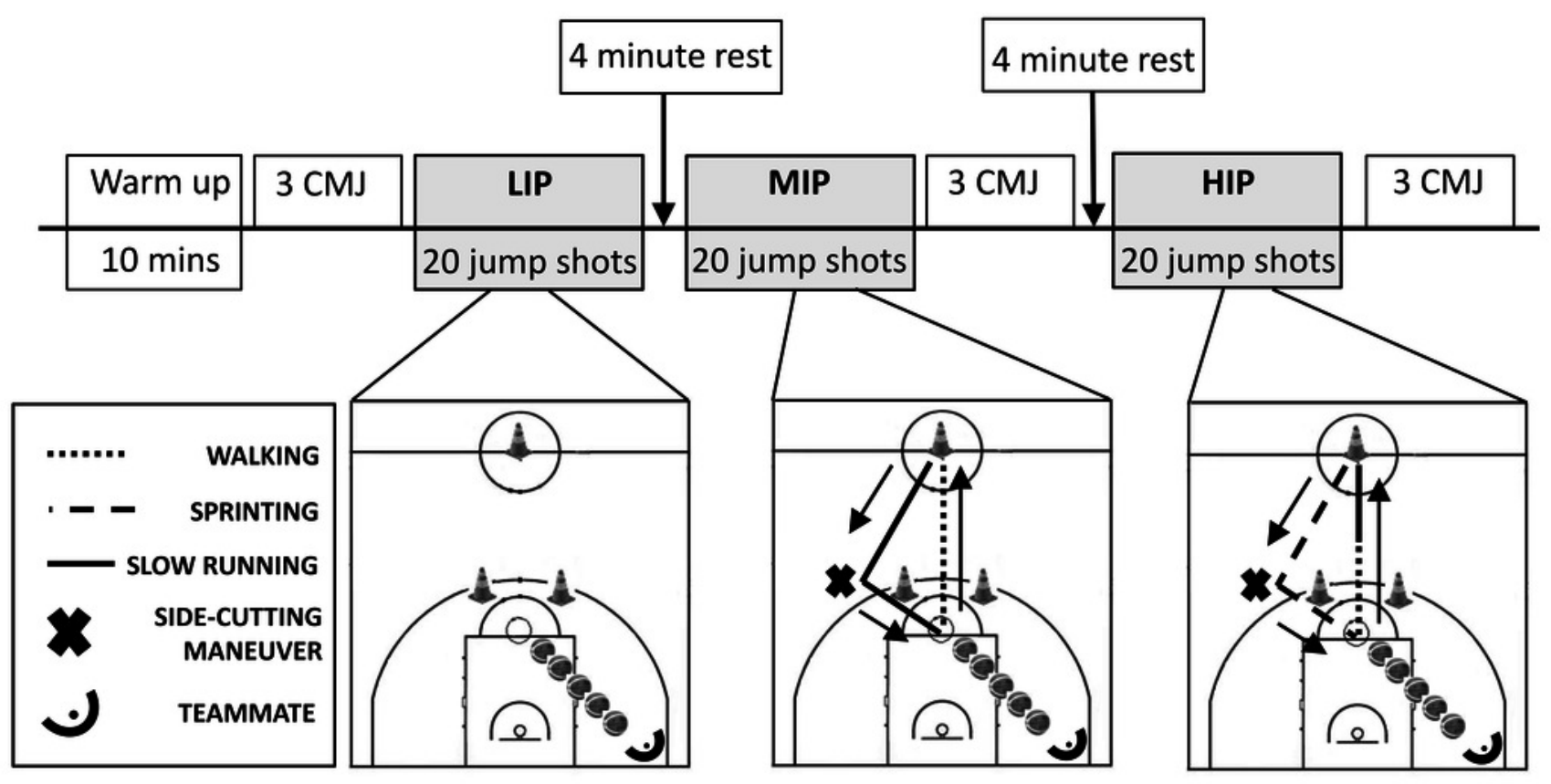


Figure 2

Jump shot accuracy

Baskets scored in the LIP (A), MIP (B) and HIP (C) conditions: comparison between experts and young players $(* p<0.05 ; * * p<0.01 ; * * * p<0.001)$.
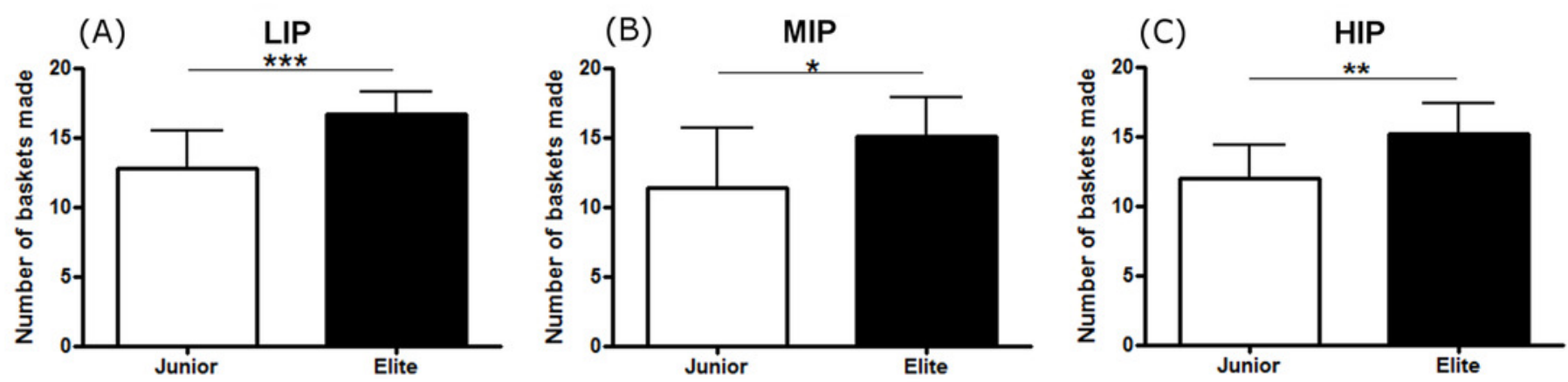


\section{Table $\mathbf{1}$ (on next page)}

Different intensities of basketball drills affect jump shot accuracy of expert and junior players. Heart rate, rate of perceived exertion (RPE), jump shot accuracy and jump height data.

Heart rate, rate of perceived exertion (RPE), jump shot accuracy and jump height recorded immediately after each fatigue protocol. Data are presented as mean $\pm \mathrm{s}$. For both groups: * different from low intensity fatigue protocol (LIP); § different from moderate intensity fatigue protocol (MIP). 


\section{Fatigue protocol}

\begin{tabular}{lccc}
\hline & LIP & MIP & HIP \\
\hline Heart Rate (beats $\cdot \mathbf{m i n}^{-\mathbf{1}}$ ) & & & $154 \pm 9 * \S$ \\
Expert players & $116 \pm 14$ & $129 \pm 11^{*}$ & $165 \pm 9 * \S$ \\
Junior players & $134 \pm 16$ & $145 \pm 11^{*}$ & \\
Karvonen Heart Rate reserve (\%) & & & $75 \pm 5^{*} \S$ \\
Expert players & $47 \pm 9$ & $57 \pm 7^{*}$ & $79 \pm 7 * \S$ \\
Junior players & $57 \pm 12$ & $65 \pm 9 *$ & $13.5 \pm 1.1 * \S$ \\
Rating of Perceived Exertion (6-20) & & & $14.4 \pm 1.3 * \S$ \\
Expert players & $8 \pm 1.5$ & $10.2 \pm 1.3 *$ & $11.2 \pm 1.2 *$ \\
Junior players & $9.8 \pm 1.3$ & & $15.2 \pm 2.2$ \\
Jump shot accuracy (baskets made) & & $15.1 \pm 2.8$ & $12 \pm 2.4$ \\
Expert players & $16.6 \pm 1.6$ & $11.4 \pm 4.2$ & $46.9 \pm 3.4$ \\
Junior players & $12.8 \pm 2.7$ & & \\
Jump height (cm) & & $49.8 \pm 3.5$ & $5.7 \pm 3.8$ \\
Expert players & & & \\
Junior players & $49.1 \pm 3.4$ & & \\
\hline
\end{tabular}


Table 2 (on next page)

Different intensities of basketball drills affect jump shot accuracy of expert and junior players. Expert and Junior shoot performance

Baskets scored for each level of induced fatigue. Experts: * Different from 1-10 series. 


\begin{tabular}{lcccccc}
\hline & \multicolumn{2}{c}{ LIP } & \multicolumn{2}{c}{ MIP } & \multicolumn{2}{c}{ HIP } \\
\hline Series of shot & $\mathbf{1 - 1 0}$ & $\mathbf{1 1 - 2 0}$ & $\mathbf{1 - 1 0}$ & $\mathbf{1 1 - 2 0}$ & $\mathbf{1 - 1 0}$ & $\mathbf{1 1 - 2 0}$ \\
\hline Groups & & & & & & \\
Expert players & $8.5 \pm 1$ & $8.1 \pm 1.1$ & $7.6 \pm 1.7$ & $7.5 \pm 1.7$ & $7 \pm 1.8$ & $8.2 \pm 0.6^{*}$ \\
Junior players & $6.3 \pm 2$ & $6.5 \pm 1.6$ & $5.5 \pm 2.4$ & $5.9 \pm 2.2$ & $5.9 \pm 1.3$ & $6.1 \pm 1.5$ \\
\hline
\end{tabular}

1 